\title{
Observation of stress relaxation via step formation in Co/Pt metallic superlattices
}

\author{
Sung-Chul Shin ${ }^{\text {a) }}$ and Young-Seok Kim \\ Department of Physics and Center for Nanospinics of Spintronic Materials, Korea Advanced Institute of \\ Science and Technology, Taejon 305-701, Korea \\ Hong-Seung Kim and Jeong-Yong Lee \\ Department of Materials Science and Engineering, Korea Advanced Institute of Science and Technology, \\ Taejon 305-701, Korea
}

(Received 4 June 1999; accepted for publication 15 December 1999)

\begin{abstract}
We report the direct observation of stress relaxation in lattice-mismatched $\mathrm{Co} / \mathrm{Pt}$ superlattices with increasing Co-layer thickness, via in situ and real-time measurements using a submonolayer-sensitive stress measurement apparatus. A large tensile stress in the Co layers found to be relaxed when the Co layer thickness was larger than 2 monolayers, corresponding to the critical thickness expected for coherent-to-incoherent transition in this system. Cross-sectional high-resolution transmission electron microscopy studies revealed that stress relaxation was realized by formation of steps, rather than misfit dislocations, near the interfaces. (C) 2000 American Institute of Physics. [S0003-6951(00)01607-7]
\end{abstract}

Stress and stress-relaxation mechanisms in heteroepitaxial thin films and multilayers are the subject of significant current interest from both the fundamental understanding of thin film growth morphology and properties, and structural stability of the thin films for technological applications. ${ }^{1}$ When the lattice misfit between the constituent film layers and/or between the film and substrate are completely absorbed by the strain energy, pseudomorphic growth, with coherent and flat interfaces, is realized. However, for thick layers and/or large lattice misfit, the strain energy becomes so large that pseudomorphic growth is no longer energetically favored and thus, some form of stress relief must occur.

The classical work of van der Merwe $\mathrm{e}^{2,3}$ and Matthews and Blakeslee ${ }^{4,5}$ has shown that the formation of misfit dislocations is a primary mechanism for relieving misfit strain in heteroepitaxial growth, when the "critical thickness" is exceeded. On the other hand, it has been recognized for several years that strained epitaxial layers can be relaxed via strain-induced surface roughening without any formation of dislocations. Tersoff and LeGoues ${ }^{6}$ have shown that small misfit strains are relaxed by the nucleation of dislocations before the surface has time to roughen, while relaxation via surface roughening is kinetically favorable at larger misfit. Thus, the surface would roughen before any dislocations are formed. Roughening by formation of up and down steps has been predicted to be the most energetically favorable arrangement. ${ }^{7} \mathrm{Ge}_{0.5} \mathrm{Si}_{0.5}$ films under compressive strains believed to form rough surface composed of single and double steps, which is energetically more stable than a flat surface. ${ }^{8}$ However, despite the general consensus that step formation is energetically favorable in surface roughening, no direct experimental evidence for stress relaxation by formation of steps has yet been reported.

In this letter, we report the real-time measurements of the stress relaxation kinetics due to step formation in a me-

a)Electronic mail: shin@kaist.ac.kr tallic system of $\mathrm{Co} / \mathrm{Pt}$ superlattices. $\mathrm{Co} / \mathrm{Pt}$ superlattices have a large misfit of $9.8 \%$ between the two constituent layers of $\mathrm{Co}(0001)$ and $\mathrm{Pt}(111)$. Thus, in the coherent regime, large tensile and compressive stresses are expected to exist in the Co and Pt layers, respectively. Therefore, with increasing layer thickness, one could expect stress relaxation via interface roughening before any nucleation of dislocations. Stress relaxation in $\mathrm{Co} / \mathrm{Pt}$ superlattices is investigated using a submonolayer-sensitive in situ stress measurement apparatus to quantify the stress relaxation in real time, together with $e x$ situ high-resolution transmission electron microscope (HRTEM) for direct observation of the growth morphology near the interfaces.

$\mathrm{Co} / \mathrm{Pt}$ superlattices were prepared by sequential dc magnetron sputtering onto glass substrates of $4 \mathrm{~cm}(l)$ $\times 1.1 \mathrm{~cm}(w) \times 130 \mu \mathrm{m}(t)$ at ambient temperature under an Ar sputtering pressure of 2 mTorr. The samples with the varying Co layer thicknesses of 4-13.2 $\AA$ (2-6 ML), with the same Pt layer thickness of $13.5 \AA$ (6 ML) and the same number of repeats of 10 , were prepared to focus our study on the effect of the Co layer thickness on the stress relaxation mechanism. The samples will be designated by $\left(\mathrm{Co}_{m} / \mathrm{Pt}_{n}\right)_{10}$, where $m$ and $n$ are the numbers of Co and Pt monolayers in a repeat distance. To study the stress relaxation kinetics we set up an in situ stress-measurement apparatus utilizing a homemade optical displacement sensing probe composed of 40 multimode optical fibers of $50 \mu \mathrm{m}$ core diameter. The apparatus could measure stress in real time every $50 \mathrm{~ms}$ during the deposition of the $\mathrm{Co} / \mathrm{Pt}$ superlattices. Details of the system have been described elsewhere. ${ }^{9}$

In Fig. 1, we show typical real-time plots of in situ measurements of the gap distance between the substrate and the optical probe as a function of the deposition time during the fabrication of two samples, comprised of $\left(\mathrm{Co}_{2} / \mathrm{Pt}_{6}\right)$ and $\left(\mathrm{Co}_{6} / \mathrm{Pt}_{6}\right)$ superlattices. Note that the displacement curves are highly reproducible in all cases and the positive and negative slopes exactly repeat every bilayer period, except 


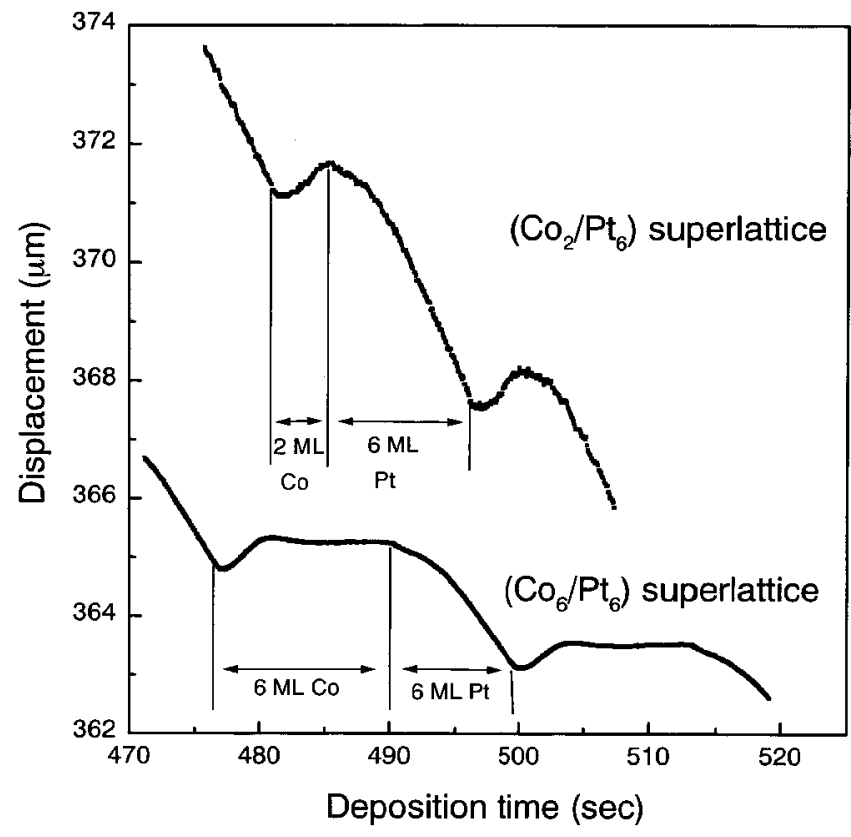

FIG. 1. Typical real-time plots of in situ measurements of the gap distance between the substrate and the optical probe with deposition time during the fabrication of $\left(\mathrm{Co}_{2} / \mathrm{Pt}_{6}\right)$ and $\left(\mathrm{Co}_{6} / \mathrm{Pt}_{6}\right)$ superlattices.

the first few bilayers due to the effect of the glass substrate. Here, the positive and negative slopes imply the existence of tensile and compressive stresses, respectively. Using the well-known Stoney's formula ${ }^{10}$ we can estimate the average stress existing in each $\mathrm{Co}$ and $\mathrm{Pt}$ layer in the $\left(\mathrm{Co}_{2} / \mathrm{Pt}_{6}\right)_{10}$ superlattice. In Fig. 2, we plot stress as a function of the Co layer. Except for the first few layers, the $\left(\mathrm{Co}_{2} / \mathrm{Pt}_{6}\right)_{10}$ superlattice reveals a constant tensile stress of 1.74 $\times 10^{10}$ dyne $/ \mathrm{cm}^{2}$ in the Co layers. Contrastively, a compressive stress of $9.57 \times 10^{10} \mathrm{dyne} / \mathrm{cm}^{2}$ in the Pt layers was measured. The stress in a superlattice film has two main origins: the adhesion of the film to the substrate and the lattice misfit between two dissimilar adjacent layers. The stress can then be deconvoluted into a substrate interaction stress and a coherency stress. ${ }^{11}$ The variation of the stress with the layer thickness in Fig. 2 implies that the substrate yields a compressive stress of $2.54 \times 10^{10}$ dyne $/ \mathrm{cm}^{2}$ in the very first Co layer, mainly due to the smaller thermal expansion coefficient of the glass substrate compared to $\mathrm{Co} / \mathrm{Pt}$ superlattice. This influence is rapidly decreased and becomes negligible in the layers after about 4 repeats or about $70 \AA$ A. Therefore, the constant tensile and compressive stresses observed in the Co and Pt layers, respectively, are considered to be mainly caused by the lattice misfit of the two constituent layers in the superlattice structure, which reset the coherent growth of Co on Pt every bilayer period.

Interestingly, the stress relaxation was always observed in $\left(\mathrm{Co}_{m} / \mathrm{Pt}_{6}\right)_{10}$ superlattices when the Co layer was thicker than $2 \mathrm{ML}$, as demonstrated for a typical example of a $\left(\mathrm{Co}_{6} / \mathrm{Pt}_{6}\right)_{10}$ superlattice sample as shown in Fig. 1. In comparison with a $\left(\mathrm{Co}_{2} / \mathrm{Pt}_{6}\right)_{10}$ superlattice, this sample shows a quite different stress dependence on the Co-layer thickness: it develops a constant tensile stress in the Co layer when thinner than about $2 \mathrm{ML}$, but the stress is completely relaxed when the Co layer is thicker than about $2 \mathrm{ML}$. The heteroepitaxy of Co on $\mathrm{Pt}$ is mainly governed by the large lattice Downloaded 23 Nov 2009 to 143.248.8.82. Redistribution subject

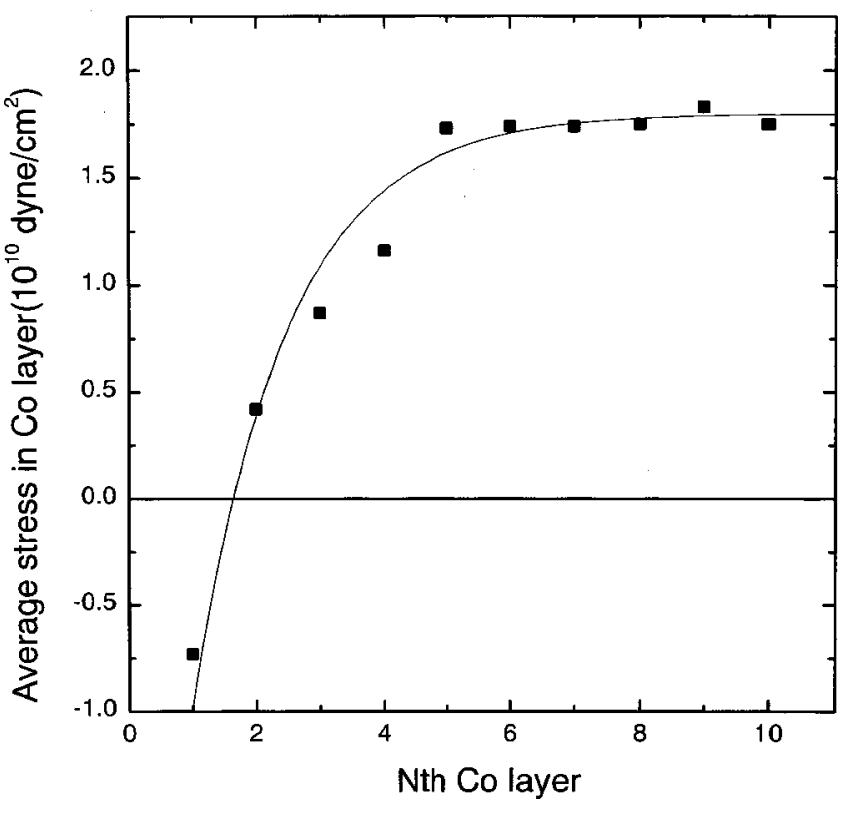

FIG. 2. Stress as a function of the Co layer in a $\left(\mathrm{Co}_{2} / \mathrm{Pt}_{6}\right)$ superlattice.

mismatch of $9.8 \%$ derived from the elemental in-plane $d$ spacings of $\mathrm{Co}$ and Pt. As a consequence of this mismatch only a few ML Co will grow coherently on Pt. Together with the observations of the $\left(\mathrm{Co}_{2} / \mathrm{Pt}_{6}\right)_{10}$ superlattice, our results clearly reveal that only the first 2 ML Co grows pseudomorphically on Pt layer, and that subsequently, stress relaxation occurs beyond this thickness via morphological evolution.

To examine how stress evolves with the deposition of Co, we calculate stress as a function of thickness of the Co layer in the $\left(\mathrm{Co}_{6} / \mathrm{Pt}_{6}\right)_{10}$ superlattice from the curve in Fig. 1. Figure 3 shows the real-time stress evolution as a function of deposition of Co on 6-ML-thick Pt layer measured every 50 $\mathrm{ms}$. Here, the data are obtained by averaging the stress generated in the Co layer of every bilayer and the error bar

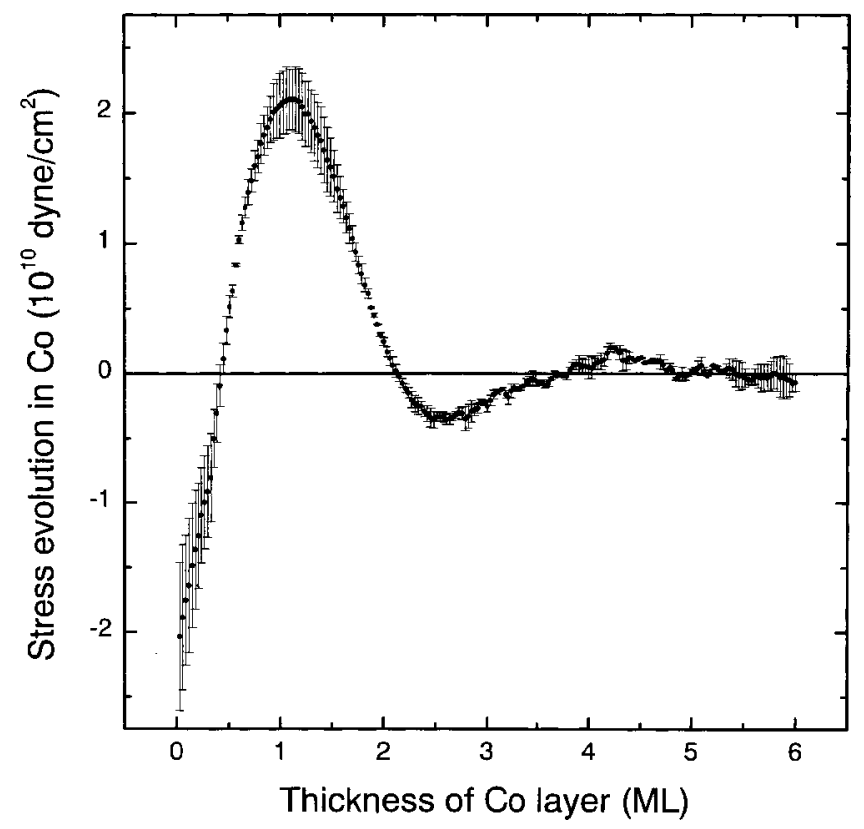

FIG. 3. Stress evolution measured in real time during deposition of Co up to $6 \mathrm{ML}$ on Pt layer. The error bars correspond to variation of stress among different bilayers. 

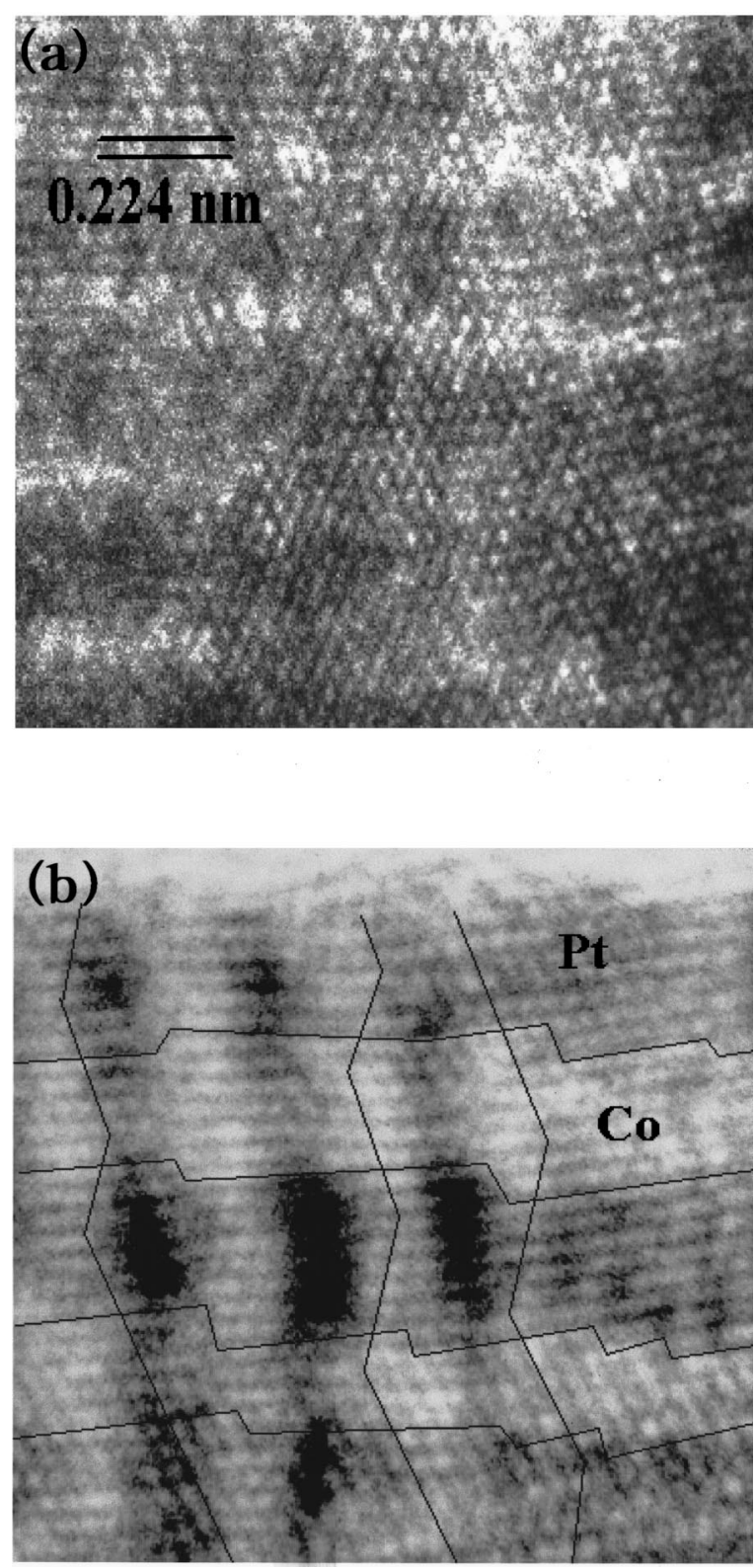

FIG. 4. Cross-sectional TEM images of (a) $\left(\mathrm{Co}_{2} / \mathrm{Pt}_{6}\right)_{10}$ superlattice and (b) $\left(\mathrm{Co}_{6} / \mathrm{Pt}_{6}\right)_{10}$ Superlattice. Steps and twins are indicated by the lines for visualization. Normal to the film plane is [110] orientation.

corresponds to the variation of stress of the Co layers in the different bilayers. Care was taken to get rid of the substrate influence on film stress by excluding the first four Co layers in averaging the data. It can clearly be seen from Fig. 3 that tensile stress in the Co layer deposited on Pt layer is nearly relieved when the thickness of Co become larger than 2 ML. A gradual change of stress into the relaxed state is evidence that the stress relaxation process in this system is kinetically limited; otherwise, an abrupt change would be observed. A small oscillatory feature in the stress relaxation at the Colayer thickness larger than 2 ML in Fig. 3 is very interesting, but it needs to be further clarified whether it originates from a real morphological evolution or an experimental error.

An interesting question to be addressed is what kind of stress relaxation mechanism is involved in $\mathrm{Co} / \mathrm{Pt}$ superlattices with increasing the Co-layer thickness. No evidence of island formation up to six monolayers of Co was observed and the Co layer was believed to grow as a two-dimensional (2D) wetting layer. Therefore, a possibility of stress relaxation via 3D islanding ${ }^{12,13}$ is unimaginable, and the formation of misfit dislocations and/or strain induced interface roughening is conceivable as the stress relaxation mechanisms in $\mathrm{Co} / \mathrm{Pt}$ superlattices. To clarify this, we have investigated the interfacial structures of the superlattice samples using cross-sectional HRTEM. Cross-sectional HRTEM specimens were prepared via mechanical polishing followed by ion milling, utilizing a specimen stage cooled by liquid nitrogen. TEM specimens were examined using a JEOL JEM 2000EX electron microscope with a point-to-point resolution of $0.21 \mathrm{~nm}$, operated at $200 \mathrm{kV}$. In Fig. 4, we show the cross-sectional HRTEM images of two Co/Pt superlattice samples revealing very different interfacial structures. Co and Pt layers can be distinguished by contrast: Pt layers are darker than Co layers due to the difference in their atomic scattering factors. The $\left(\mathrm{Co}_{2} / \mathrm{Pt}_{6}\right)_{10}$ superlattice shown in Fig. 4(a) reveals that the matching planes are in relatively good lattice registry at the interfaces between Co and Pt layers. In contrast, we observe many steps and twins in the Co and $\mathrm{Pt}$ layers in the $\left(\mathrm{Co}_{6} / \mathrm{Pt}_{6}\right)_{10}$ superlattice sample, as beautifully demonstrated in Fig. 4(b). We did not observe any interfacial misfit dislocations in the superlattice samples having Co layers thicker than the critical thickness. Therefore, it could be claimed that stress relaxation observed in Fig. 2 is realized by the introduction of interfacial steps. Finally, we like to mention that the TEM results were consistent with $\theta-2 \theta$ scanning x-ray diffraction studies: only one major Bragg peak, close to the $d$ spacing of bulk $\operatorname{Pt}(111)$, was observed for the $\left(\mathrm{Co}_{2} / \mathrm{Pt}_{6}\right)_{10}$ sample, but two peaks were observed for the $\left(\mathrm{Co}_{6} / \mathrm{Pt}_{6}\right)_{10}$ sample.

This work was supported by the Creative Research Initiatives and Nanostructure Technology Project of the Korean Ministry of Science and Technology. The authors are grateful to N.-Y. Bae for her help with HRTEM studies.

${ }^{1}$ S. C. Jain, A. H. Harker, and R. A. Cowley, Philos. Mag. A 75, 1461 (1997), and references therein.

${ }^{2}$ J. H. van der Merwe, J. Appl. Phys. 34, 117 (1963).

${ }^{3}$ J. H. van der Merwe, J. Appl. Phys. 34, 123 (1963).

${ }^{4}$ J. W. Matthews and A. E. Blakeslee, J. Cryst. Growth 29, 273 (1975).

${ }^{5}$ J. W. Matthews and A. E. Blakeslee, J. Cryst. Growth 32, 265 (1976).

${ }^{6}$ J. Tersoff and F. K. LeGoues, Phys. Rev. Lett. 72, 3570 (1994).

${ }^{7}$ J. Tersoff, Phys. Rev. Lett. 74, 4962 (1995).

${ }^{8}$ Y. H. Xie, G. H. Gilmer, C. Roland, P. J. Silverman, S. K. Buratto, J. Y. Cheng, E. A. Fitzgerald, A. R. Kortan, S. Schuppler, M. A. Marcus, and P. H. Citrin, Phys. Rev. Lett. 73, 3006 (1994).

${ }^{9}$ Y.-S. Kim and S.-C. Shin, Thin Solid Films 258, 128 (1995).

${ }^{10}$ G. G. Stoney, Proc. R. Soc. London, Ser. A 82, 172 (1909).

${ }^{11}$ J. A. Bain, L. J. Chyung, S. M. Brennan, and B. M. Clemens, Phys. Rev. B 44, 1184 (1991).

${ }^{12}$ B. J. Spencer, P. W. Voorhees, and S. H. Davis, Phys. Rev. Lett. 67, 3696 (1991).

${ }^{13}$ J. A. Floro, E. Chason, R. D. Twesten, R. Q. Hwang, and L. B. Freund, Phys. Rev. Lett. 79, 3946 (1997). 